This item was submitted to Loughborough's Research Repository by the author.

Items in Figshare are protected by copyright, with all rights reserved, unless otherwise indicated.

\title{
Mask-free laser lithography for rapid and low-cost microfluidic device fabrication
}

PLEASE CITE THE PUBLISHED VERSION

https://doi.org/10.1021/acs.analchem.8b03169

\section{PUBLISHER}

(C) American Chemical Society

\section{VERSION}

AM (Accepted Manuscript)

\section{PUBLISHER STATEMENT}

This document is the Accepted Manuscript version of a Published Work that appeared in final form in Analytical Chemistry, copyright $\odot$ American Chemical Society after peer review and technical editing by the publisher. To access the final edited and published work see https://pubs.acs.org/doi/10.1021/acs.analchem.8b03169.

\section{LICENCE}

CC BY-NC-ND 4.0

\section{REPOSITORY RECORD}

Trantidou, Tatiana, Mark S. Friddin, Kin B. Gan, Luyao Han, Guido Bolognesi, Nicholas J. Brooks, and Oscar Ces. 2019. "Mask-free Laser Lithography for Rapid and Low-cost Microfluidic Device Fabrication". figshare. https://hdl.handle.net/2134/36851. 
This document is confidential and is proprietary to the American Chemical Society and its authors. Do not copy or disclose without written permission. If you have received this item in error, notify the sender and delete all copies.

\section{Mask-free laser lithography for rapid and low-cost microfluidic device fabrication}

\begin{tabular}{|r|l|}
\hline Journal: & Analytical Chemistry \\
\hline Manuscript ID & ac-2018-03169g.R1 \\
\hline Manuscript Type: & Article \\
\hline Date Submitted by the & $\mathrm{n} / \mathrm{a}$ \\
\hline Complete List of Authors: & $\begin{array}{l}\text { Trantidou, Tatiana; Imperial College London, Chemistry } \\
\text { Friddin, Mark; Imperial College London, Chemistry } \\
\text { Gan, Kin; Imperial College London } \\
\text { Han, Luya; Imperial College London, Chemistry } \\
\text { Bolognesi, Guido; Loughborough University, Chemical Engineering } \\
\text { Brooks, Nicholas; Imperial College London, Department of Chemistry } \\
\text { Ces, Oscar; Imperial College, Department of Chemistry }\end{array}$ \\
\hline &
\end{tabular}

\section{SCHOLARONE ${ }^{\text {W }}$ Manuscripts}


Microfluidics has matured into a well-established and versatile field with a myriad of applications spanning the life sciences. ${ }^{1,2}$ With the potential for this platform technology gradually being realized, there has never been greater demand for quick, simple and low-cost alternatives to soft lithography, an approach that has dominated chip fabrication for over two decades. While this term was originally used to group several techniques where an elastomeric stamp or mould is used to transfer a pattern to a substrate, ${ }^{3,4}$ in recent years soft lithography has typically referred to the use of a photoresist patterned silicon or glass wafer as a master for casting the widely used elastomer poly(dimethylsiloxane) (PDMS). Although this approach ensures the reliable fabrication of micro-structured devices in a material that has been well characterised, ${ }^{5}$ the process is time consuming, requires a photomask, expensive consumables and inevitably involves specialist training when being performed inside a cleanroom environment. In addition, any slight modification to the design of the microfluidics requires the whole process to be repeated, leading to significant delays between device iterations.

To address this problem, several alternatives to device fabrication have emerged in recent years. This includes a range of different additive manufacturing techniques capable of producing fully enclosed chips equipped with customized interconnects. ${ }^{6}$ Driven by the ever-reducing cost of printers, the increasing availability of materials and continual improvements in print resolution, additive manufacturing has been shown to be an excellent alternative to soft lithography, ${ }^{7,8}$ however, it too suffers from significant and often understated drawbacks. These include the difficulty of removing uncured resin from microchannels, limited control in terms of surface functionalization and wettability (particularly when it is not possible to pause printing), and the often cumbersome requirement to optimize the printing conditions. ${ }^{9}$

Another alternative to soft lithography for manufacturing microfluidic devices is the use of dry resist films (DRF). Compared to SU-8, these photosensitive sheets offer higher planarity over large substrate areas, ${ }^{10}$ shorter processing times and the ability to laminate multiple sheets together - a process that would typically require multiple spin-coating and soft baking cycles when using SU-8. The technique also eliminates the need to silanize wafers and prepare degassed PDMS, while hugely simplifying any downstream bonding steps. To this end DRFs have been widely used in microfluidic device fabrication as masks for wet-etching glass, ${ }^{11}$ as masters for soft lithography ${ }^{12}$ and as stamps for hot embossing, ${ }^{13,14}$ in addition to forming hybrid master templates using SU-8 to manufacture PDMS multi-layered devices. ${ }^{15}$ In applications where interfacing microfluidic devices with electronic components is essential, DRFs have also been leveraged to structure microfluidic channels for $\mu \mathrm{PCR}^{16,17}$ and electrophoretic actuation applications. ${ }^{18-20}$ Yet despite these 
advantages, DRF in all these applications still require patterning using a mask and a UV light-source and thus require centralized fabrication facilities for device preparation.

Here we report a new approach that eliminates these requirements entirely, enabling microfluidic chips to be fabricated outside of the cleanroom in less than 15 minutes. We achieve this using a standard flatbed laser to directly cut channels into sheets of poly(methyl methacrylate) (PMMA)

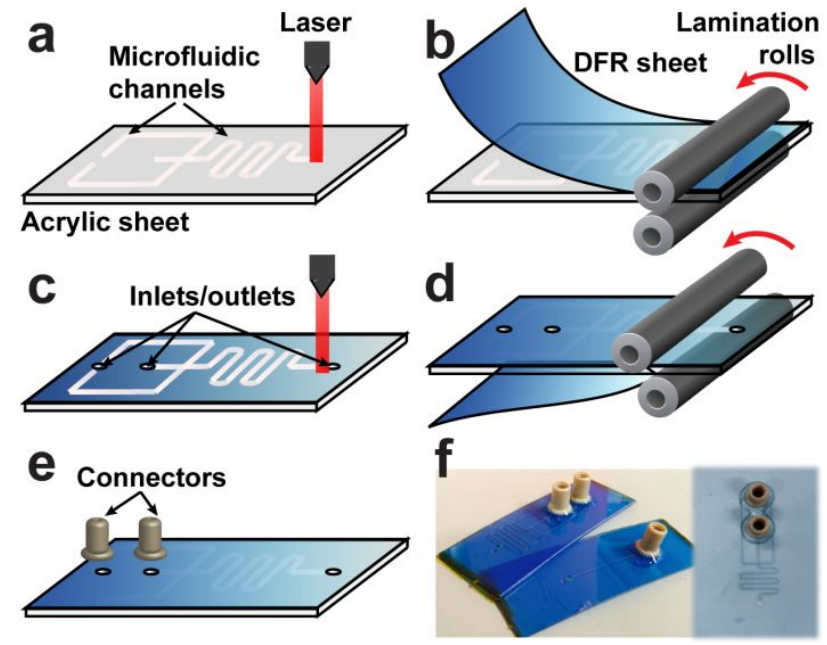

Fig. 1 (a) A PMMA sheet is laser cut to create the microfluidic channels. (b) A DRF sheet is laminated on the PMMA sheet using lamination rolls. (c) The device is flipped over and inlets and outlets are laser cut. (d) A second DRF lamination seals the microchannels and inlets/outlets. (e) Interfacing connectors are placed on the inlets. (f) Prototyped microfluidic devices and close up of the microfluidic channel pattern.

that are subsequently laminated with DRF. Our technique is totally mask-free, does not require UV exposure, requires only a standard flatbed laser cutter and significantly deskills device fabrication. We demonstrate that chips engineered using our method, which we term Laser Lithography (LL), can be used to generate monodisperse microdroplets in dual-phase systems from either surfactant or lipids, and show that these can be engineered reproducibly and comprised of two aqueous components capable of supporting a chemical reaction onchip. The versatility of our approach, namely the ability to produce any given microfluidic geometry almost immediately, is further enhanced by demonstrating the compatibility of our devices with a synchrotron light-source, meaning that our technique could be used for applications requiring short- or wide- angle X-ray scattering (SAX/WAX). Our results represent a significant step toward democratizing the fabrication of microfluidic chips, further encouraging the interdisciplinary uptake of microfluidics as a paradigmshifting platform technology through a new generation of complex rapid prototyped devices.

\section{EXPERIMENTAL}

Microfluidic device fabrication. PMMA sheets $(0.2 \mathrm{~mm}$ thick) were purchased from Weatherall (Wendover, UK). The protective sheets were removed and the acrylic sheets were cut using a VLS660 flatbed laser (Universal Laser Systems, USA) from a CAD or Corel Draw design to create the microfluidic channel network. The laser cut pieces were cleaned in isopropyl alcohol (IPA) and thoroughly dehydrated on a hotplate (at $110{ }^{\circ} \mathrm{C}$ for at least $15 \mathrm{~min}$ ). The PMMA sheets were laminated on one side using DRF (Ordyl SY355, MegaElectronics Ltd, Linton, UK) using an office laminator (A3 Mega Drive Laminator, MegaElectronics Ltd) at $55^{\circ} \mathrm{C}$ temperature and $1 \mathrm{~m} \cdot \mathrm{s}^{-1}$ speed. The pressure during lamination was maintained constant at approximately 90 psi. The laminated PMMA sheets were subsequently laser cut to create the inlets/outlets. The devices were again cleaned in IPA and dehydrated as described before being sealed via another lamination with DRF. Interfacing connectors $\left(\right.$ Upchurch $^{\circledR}$ Scientific NanoPort Assemblies, IDEX, London, UK) were fixed on top of the inlets/outlets using an epoxy resin (RS Components, Corby, UK). The NanoPort Assemblies ${ }^{\mathrm{TM}}\left(10^{-32}\right.$ Cnd 1/16") were connected via FEP tubing (IDEX, London, UK) to syringes controlled by syringe pumps (Fusion 200, Chemyx, Stafford, TX, USA). The fabrication process is outlined in Fig. 1a-e and examples of fully assembled devices are highlighted in Fig. 1f. All devices were stored in standard laboratory conditions prior to use.

Solutions and dispersions for droplet generation. Surfactant-stabilised droplets were generated using Span-80 (Sigma-Aldrich, Dorset, UK) in squalene (Fisher Scientific Ltd, Loughborough, UK), while the aqueous phase consisted of Tris Acetate-EDTA (TAE) buffer (Sigma-Aldrich, Dorset, $\mathrm{UK}$ ). Methylene blue hydrate and $\mathrm{Na}_{2} \mathrm{SO}_{3}$ (all Sigma-Aldrich, Dorset, UK) were used for the reduction reaction. DPhPC (diphytanoyl phosphatidylcholine) lipid powder was purchased by Avanti Polar Lipids (Alabaster, AL, USA). The desired amount of lipid was dissolved in chloroform (SigmaAldrich, Dorset, UK) and gently evaporated using a nitrogen stream to form a lipid film, which was fully dehydrated by placing in a lyophilizer overnight. TAE buffer and squalene were added to the lipid films for lipid-in and lipid-out methods respectively, and the mixture or dispersion was sonicated for $30 \mathrm{~min}$ in a benchtop ultrasonic bath.

Small angle X-ray diffraction (SAXS). Experiments were performed at Beamline I22, Diamond Light Source. Diffraction patterns were collected using an X-ray wavelength of $1 \AA$, a sample to detector distance of $4.7 \mathrm{~m}$ and a beam size of approximately $0.3 \mathrm{~mm}$ wide by $0.1 \mathrm{~mm}$ high. Devices were secured using a custom-built frame and positioned to ensure the sample channel was centered on the X-ray beam.

Data acquisition and statistical analysis of droplet size. Imaging was performed using an inverted Olympus IX81 microscope with a high-speed Phantom camera (Vision Research Ltd, Bedford, UK). Microfluidic mixing experiments were imaged with an inverted Leica DM IRB microscope (Leica Microsystems Ltd, Milton Keynes, UK). Droplet size was calculated using an automated process developed in ImageJ (NIH, Bethesda, MD, USA) and Python. The monodispersity of the droplets was extrapolated from the Gaussian fitting of the histogram as the standard deviation over the average value (coefficient of variation). When this number was smaller than $5 \%$, the droplet generation was assumed to be monodisperse. 


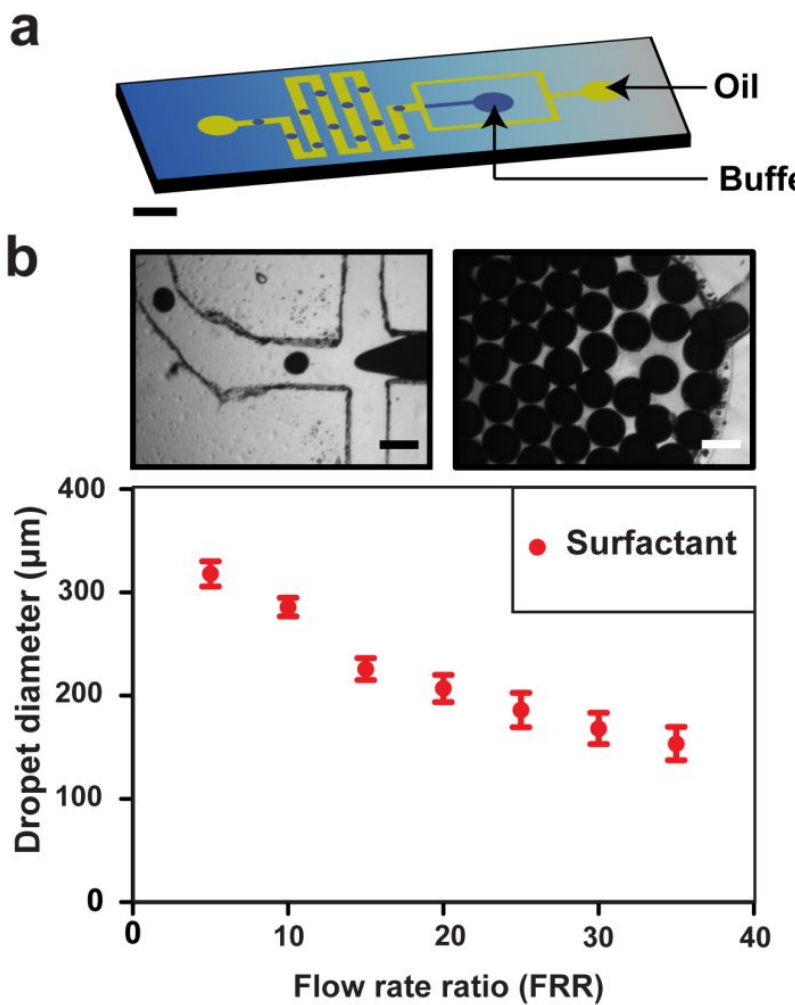

C
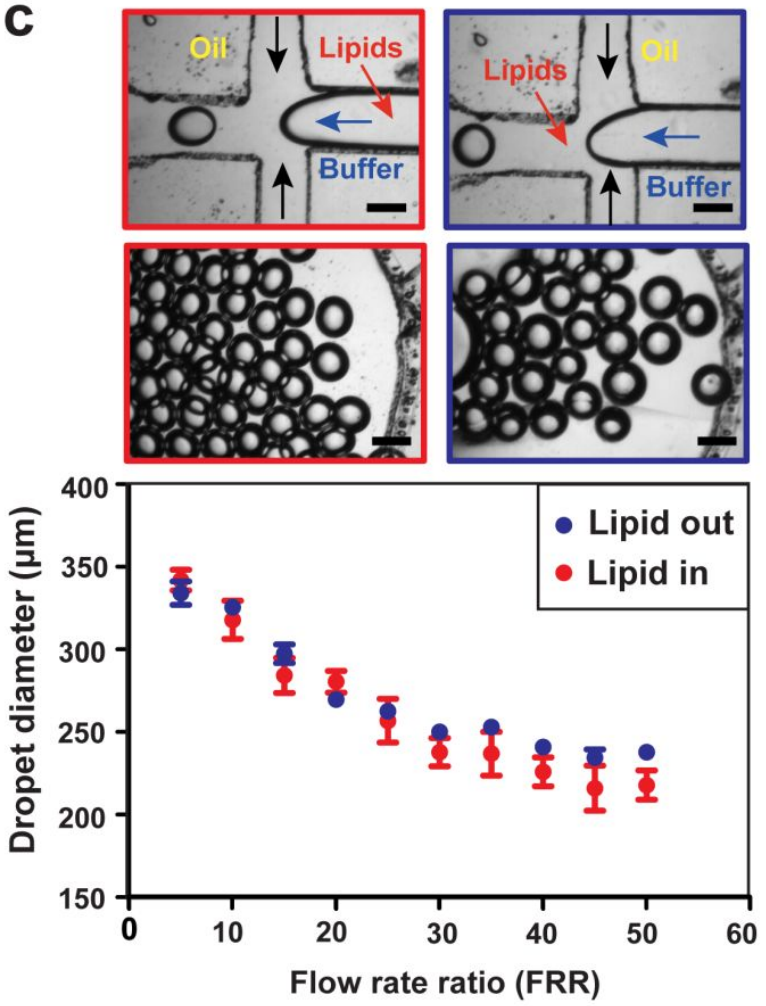

Fig. 2 (a) Schematic diagram of the prototyped microfluidic device showing squalene and TAE buffer as the continuous and disperse phases respectively. The channel cross section of the device is $200 \times 200 \mu \mathrm{m}$. Scale bar is $10 \mathrm{~mm}$. (b) Micrographs of generated droplets at FRR 20 and corresponding droplet diameter of surfactant (Span80)-stabilized droplets produced at distinct flow rate ratios. (c) Micrographs of generated droplets at FRR 20 and corresponding droplet diameter of lipidstabilized droplets produced at distinct flow rate ratios using the lipid-in and lipid-out method. Error bars correspond to the standard deviation (SD). Scale bars are $200 \mu \mathrm{m}$.

\section{RESULTS}

Microfluidic droplet generation. Devices were tested for their ability to generate stable and monodisperse water in oil (w/o) droplets. The versatility of our proposed approach was demonstrated using either anionic surfactants $(0.1 \mathrm{wt} \%$ Span80) or phospholipids (DPhPC) in the oil phase (squalene). Devices were manufactured with a flow focusing junction (Fig. 2(a)) comprising of a $200 \times 200 \mu \mathrm{m}$ cross section. The ability to control droplet size by adjusting the applied flow rate ratio (FRR) is depicted in Fig. 2(b). FRR is defined as the oil volumetric flow rate divided by the aqueous volumetric flow rate. As expected, the diameter of the generated surfactant-stabilised droplets was observed to scale inversely with FRR..$^{21,22}$ The droplets remained stable and did not fuse over time at the outlet. Furthermore, we employed DPhPC lipid as surfactant to generate lipid-stabilised w/o droplets (Fig. 2(c)). These components comprise the potential building blocks for fabricating multicompartment artificial cells..$^{23}$ To demonstrate the versatility of our approach, lipids were either supplied to the aqueous phase (lipid-in) or in the oil phase (lipid-out). $3 \mathrm{mg} \cdot \mathrm{ml}^{-1}$ of DPhPC was prepared in TAE buffer for the lipid-in experiments and $10 \mathrm{mg} \cdot \mathrm{ml}^{-1}$ of lipid was dissolved in squalene for the lipid-out experiments. Fig. 2(c) depicts micrographs of lipid-stabilised w/o droplet generation at the FFJ and at the outlet. In both approaches droplet diameter decreased inversely with increased FRR. The monodispersity of the produced droplets was low, with a coefficient of variation (CV) lower than $5 \%$ - indicating narrow size distributions in both cases. In particular, the lipidout approach demonstrated considerably lower CV than the lipid-in method with $\mathrm{CV}$ varying between 0.8 and $2.1 \%$ for all tested FRR. As expected, droplets generated using the lipid-in approach were more stable and did not fuse with other droplets at the outlet, whereas lipid-out droplets sporadically fused together, yielding larger droplets. The improved droplet stability using the lipid-in approach is attributed to the shorter incubation time required for the interfacial lipid monolayer to form at the boundary of the droplet compared to the lipid-out approach. ${ }^{24} \mathrm{We}$ also capitalized on our data addressing mean droplet size to characterize any device-to-device variation in our fabrication process. Our results (Figure S1, Supporting Information) demonstrate that there was no significant difference in performance between chips. Similarly, we also found that our results were independent of any light-driven photochemical reactions sustained by the DRF. To confirm this, we conducted experiments where devices were either exposed to collimated UV light $\left(300 \mathrm{~mJ} \mathrm{~cm}^{-2}\right)$ or left exposed to ambient laboratory light prior to microfluidic testing. Our 
results showed that there was no observable difference in terms of droplet generation between the two sets of devices (Figure S2, Supporting Information). Although we found that the light blue tint of devices faded over time, we elected to probe the optical transparency of our devices using a spectrophotometer. As maintained by the literature supplied by the manufacturer, our results showed that our devices exhibited very low absorbance in the visible spectrum, corresponding to high optical transmittance (Figure S3, Supporting Information). This finding supports our claim that our LL technology can be adopted for a wide range of applications that rely on the optical accessibility of the sample. However another urgent factor that is required to reinforce our claim is the strength of the bond between the layers comprising our devices. We characterized this force by steadily increasing the flow at the inlets of our devices and solving for the pressure drop within the device analytically (Calculation S1, Supporting Information). We found that our devices were repeatedly able to withstand a flow rate of 1 $\mathrm{ml} \cdot \mathrm{min}^{-1}$ which, given our device geometry corresponds to a pressure drop of ca. 3.3 bar between the inlet and the outlet. At higher flow rates we found that leakage typically arose due to failure of the adhesive used to bond the inlets/outlets rather than due to the delamination of devices. Finally, to address the roughness of the channels engineered using our technique, we obtained micrographs of channels and analysed them using ImageJ to determine the distance between the tallest peak and the deepest valley in each sample $\left(\mathrm{R}_{\mathrm{z}}\right)$ and the RMS of the height of the wall profile $\left(\mathrm{R}_{\mathrm{q}}\right)$ (Figure $\mathrm{S} 4$, Supporting Information). We found that $\mathrm{R}_{\mathrm{z}}$ values ranged from $3.9 \mu \mathrm{m}$ to $44.8 \mu \mathrm{m}$ while $\mathrm{R}_{\mathrm{q}}$ values ranged from $1.2 \mu \mathrm{m}$ to $8.9 \mu \mathrm{m}(\mathrm{n}=$ 26). While this degree of surface roughness is greater than what would be expected for devices engineered using soft lithography, we saw no evidence to suggest that this negatively affected our results.

Microfluidic mixing. Devices were manufactured with two aqueous inlets and one oil inlet, a flow focusing junction for droplet generation of $200 \times 200 \mu \mathrm{m}$ cross section and a serpentine with 8 turns to enable chaotic mixing along its length (Fig. 3(a)). As a proof of principle, the microfluidic devices were used to precisely control the dilution of Methylene blue dye $\left(M^{n+}\right)$ in TAE buffer inside microsized droplets. Methylene blue is a cationic dye which is extensively used in textile industry and in manufacturing colour pens and polygraphic inks. ${ }^{25,26} 10 \mathrm{mg} \cdot \mathrm{ml}^{-1}$ of $\mathrm{DPhPC}$ was dissolved in squalene, which served as the continuous phase at a constant flow rate of $20 \mu \mathrm{min}^{-1}$. The two aqueous (disperse) phases comprised of Methylene blue in TAE $\left(5 \mathrm{mg} \cdot \mathrm{ml}^{-1}\right)$ and TAE buffer, with the total volumetric flow rate (the sum of the flow rates of the two aqueous phases) always at $2 \mu \mathrm{l} \cdot \mathrm{min}^{-1}$ to maintain similar droplet sizes for all conditions. Depending on the flow rate of the dye phase, different levels of mixing and dilution were achieved with precision. We produced a calibration curve in order to extrapolate a direct correlation between greyscale levels of the acquired droplet images and the actual dye concentration in them, and we leveraged this calibration curve to relate the dye flow rate with the final concentration of Methylene blue. Fig. 3(b) depicts representative greyscale microscopy images of droplets generated with $0.1,0.6$ and $1 \mu \mathrm{l} \cdot \mathrm{min}^{-1}$ flow rate of the dye stream. Data revealed a linear correlation between the flow rate of the dye and the resulting dye concentration in the droplets at the outlet is also depicted. As mentioned above, the overall volumetric flow rate of the two aqueous inlets was
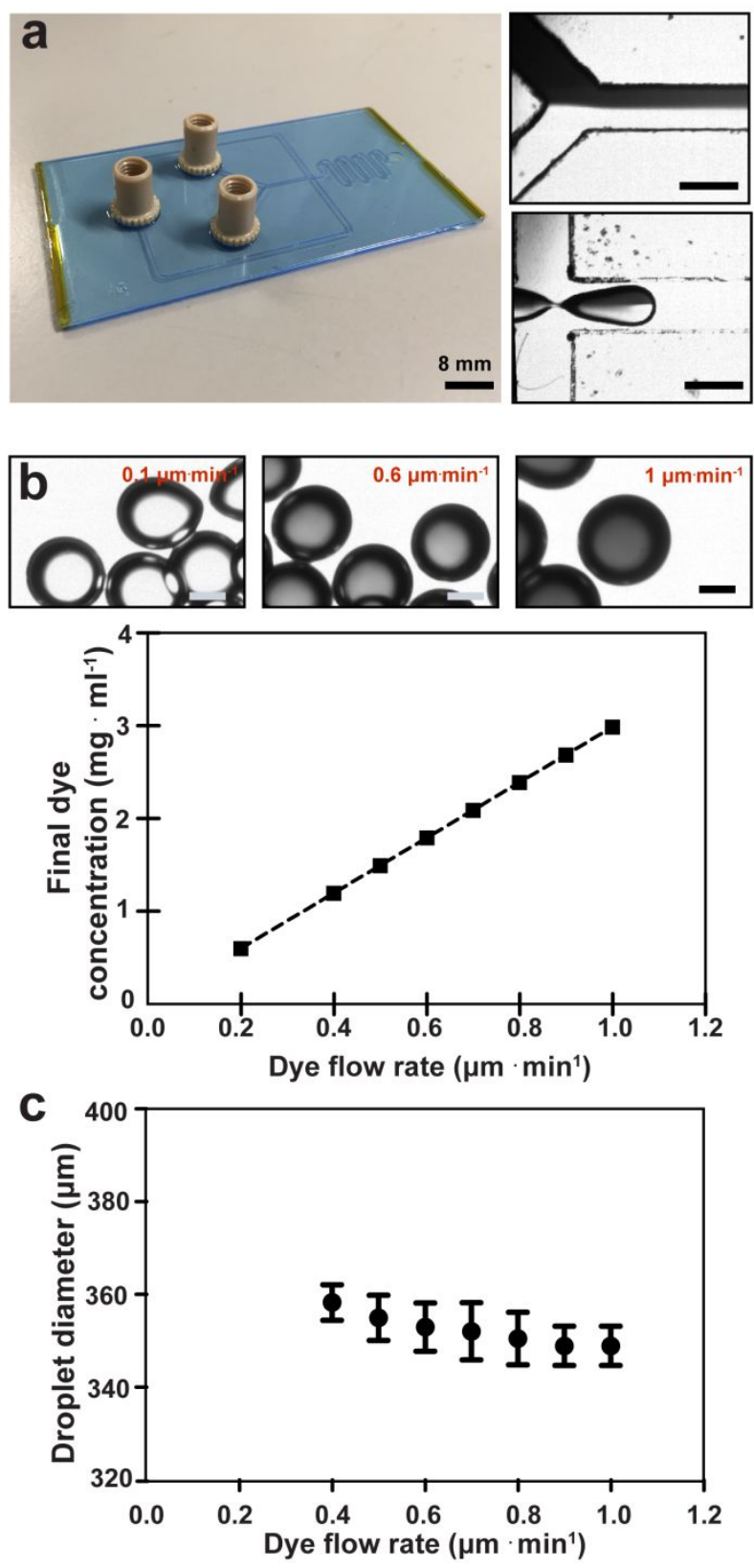

Fig. 3 (a) Prototyped microfluidic mixer chips and indicative micrographs of droplet mixing (methylene blue and buffer) at a microfluidic junction. Scale bars $200 \mu \mathrm{m}$. (b) Methylene blue concentration as a function of the dye flow rate. Scale bars are $150 \mu \mathrm{m}$. (c) Droplet diameter of lipid-stabilized droplets produced at distinct dye flow rate ratios. Error bars correspond to the standard deviation (SD).

always maintained at $2 \mu \mathrm{l} \cdot \mathrm{min}^{-1}$, which did not result in significantly different droplet sized for all dye flow rates, as shown Fig. 3(c).

As a proof-of-concept, we leveraged the microfluidic mixing device to use the droplets as microreactors, enabling the reduction of Methylene blue at room temperature. Decolourization of Methylene blue via oxidation is one of several methods available for removal of this dye from effluents. ${ }^{27}$ The proposed reaction of decolourization of Methylene blue is shown in Fig. 4(a), while the distinct chemical compounds used in this experimental setup are 
a

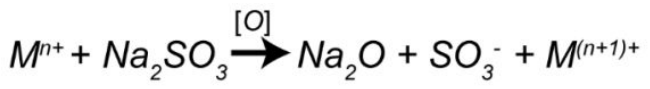

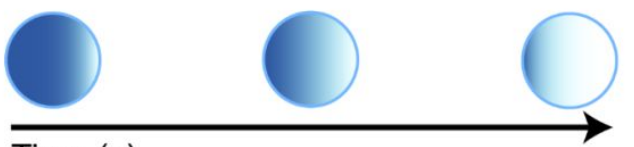

b

Time (s)
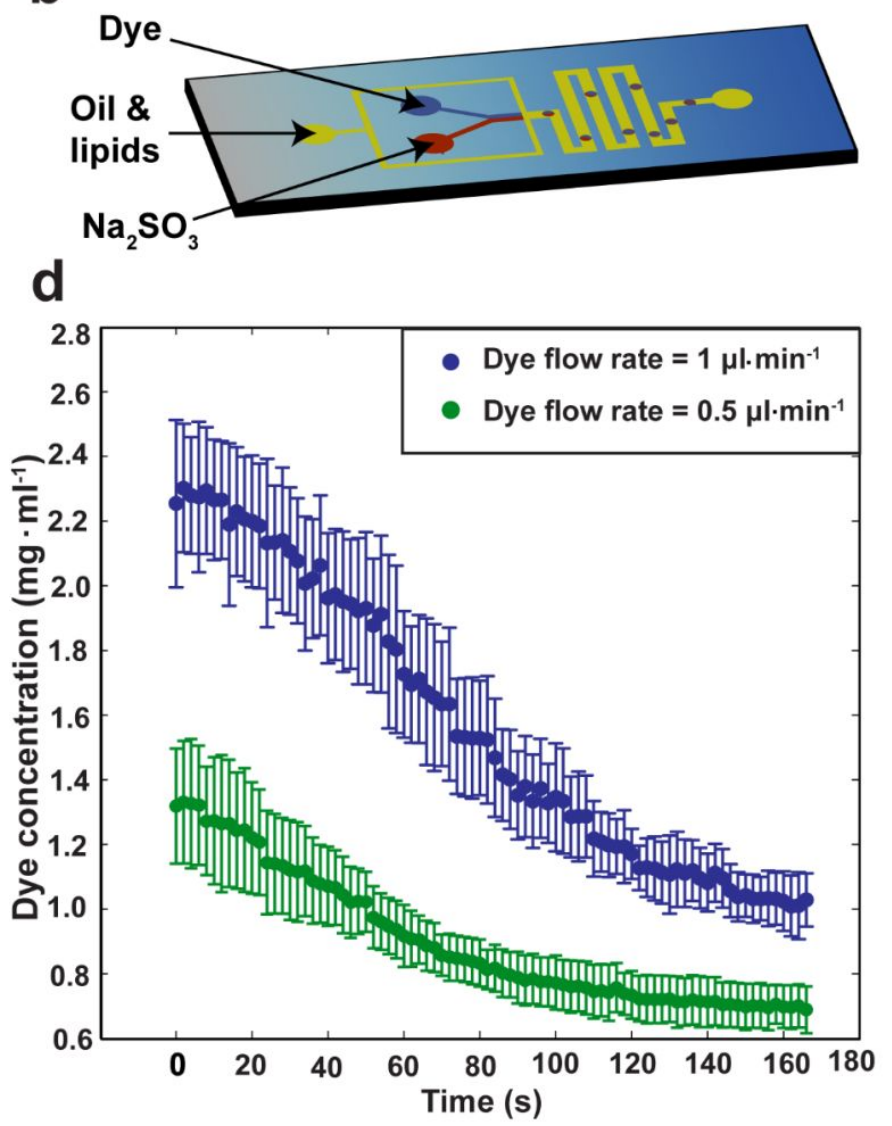

C

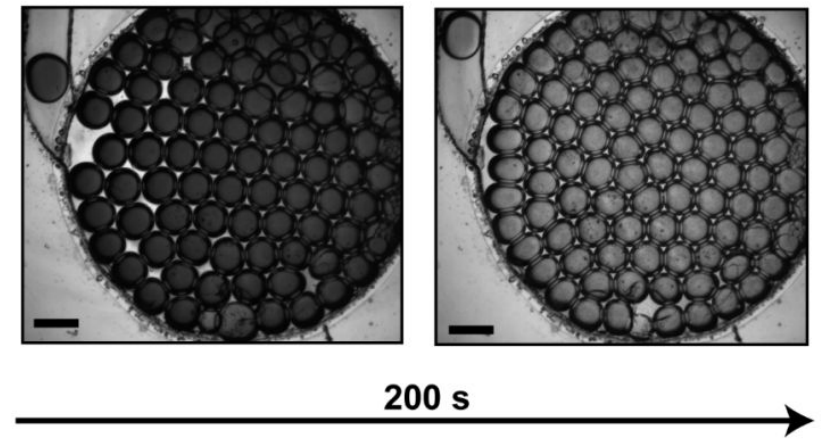

e

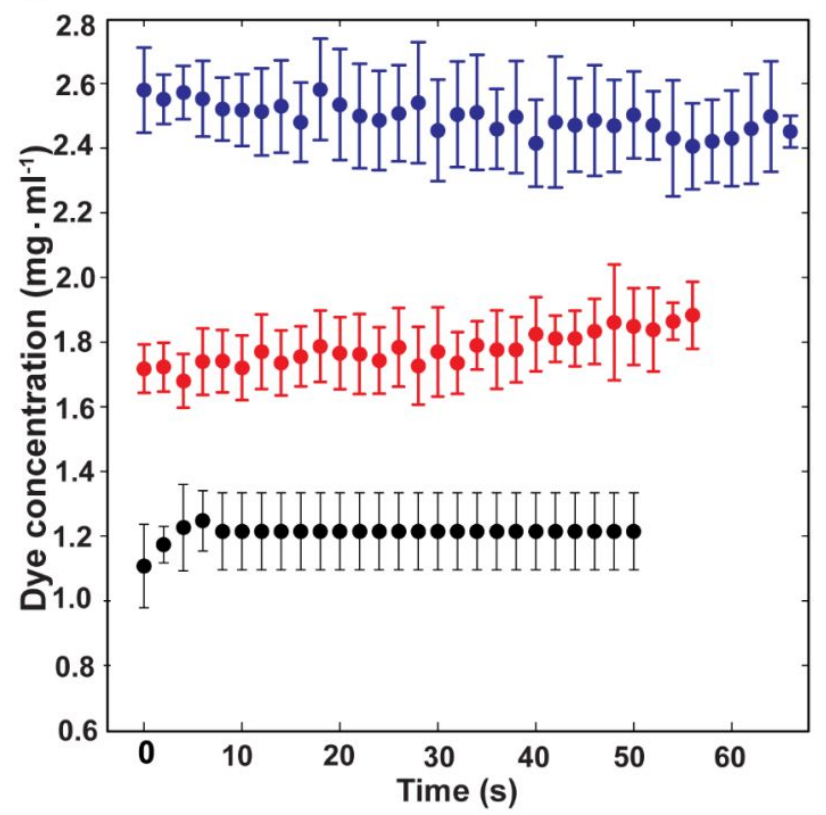

Fig. 4 (a) Proposed reaction of decolorization of Methylene blue. (b) Schematic diagram of the prototyped microfluidic mixer showing distinct chemical components. (c) Micrographs showing decolorization of Methylene blue over $200 \mathrm{~s}$. Scale bars $200 \mu \mathrm{m}$. (d) Methylene blue concentration over time for dye flow rate 1 (blue) and 0.5 (green) $\mu \mathrm{m} \cdot \mathrm{min}^{-1}$. (e) Methylene blue concentration over time in the absence of the reducing agent for dye flow rates 1 (blue), 0.6 (red) and 0.4 (black) $\mu \mathrm{m} \cdot \mathrm{min}^{-1}$. Error bars correspond to the standard deviation (SD).

shown in Fig. 4(b). Methylene blue is blue when it is in an oxidising environment, but becomes colorless (leuco Methylene blue) if it is exposed to a reducing agent. In this setup we used $1.5 \mathrm{M}$ of $\mathrm{Na}_{2} \mathrm{SO}_{3}$ in TAE buffer as the reducing agent. The dye comprised of Methylene blue in TAE (5 $\mathrm{mg} \cdot \mathrm{ml}^{-1}$ ), while the continuous phase comprised of $10 \mathrm{mg} \cdot \mathrm{ml}^{-1}$ of DPhPC in squalene to facilitate droplet formation. Fig. 4(c) demonstrates representative greyscale microscopy images of generated droplets in a time course of $200 \mathrm{~s}$. It is evident that the reaction progresses over this time, and consequently Methylene Blue decolorizes. Fig. 4(d) depicts the kinetics of decolorization of Methylene blue with sulphite in TAE buffer, showing changes in the dye concentration over time for two distinct flow rates of the dye stream, 1 and $0.5 \mu 1 \cdot \mathrm{min}^{-1}$ which further determine the dilution ratio of the dye to the reducing agent to $1: 1$ and 1:2 respectively. In the absence of the reducing agent, the concentration of Methylene blue remained unchanged over time for all distinct flow rates of the dye stream (Fig. 4(e). It is noteworthy that based on Fig. 4(d), the rate constant of the reaction is not constant, as it decreases upon increasing concentration of Methylene blue. These results are in accordance with previous studies demonstrating decolorization of Methylene Blue in bulk. ${ }^{28}$

In situ small angle X-ray diffraction (SAXs) experiments. SAX is widely used to investigate the assembly structure of soft materials. It has been coupled to traditional microfluidic technology to both allow optimization of flow rates and investigate structural changes that take place in response to specific flow fields. ${ }^{29}$ LL technology offers potential for convenient containment of soft materials under static conditions, under flow and after rapid mixing for both static and time resolved SAXS. In addition, the rapid production turnaround for such devices allows a new paradigm in microfluidic processing and flow field optimization, where the device can be optimized, in near real time, with direct engineering feedback from obtained structural data. Critically, the materials used in LL offer very little background X-ray 
scattering and low X-ray attenuation. Here, we have carried out a series of proof-of-concept SAXS experiments using microfluidic devices fabricated via LL. Fig. 5 shows the small angle X-ray diffraction pattern from a DOPC sample (30 wt $\%$ in water) pumped into a channel in the DRF microfluidic device. It can be seen that the device exhibits extremely low background scattering, which is comparable to traditional glass X-ray capillary containers, ${ }^{30}$ and was entirely stable to synchrotron X-ray exposure over at least 10 seconds per measurement point. Importantly, the channel dimensions (200 $\mu \mathrm{m} \times 200 \mu \mathrm{m})$ are significantly smaller than traditional SAXS sample containers, opening the possibility of rapid online processing and containment of scarce samples.

\section{DISCUSSION}

Our results showcase the use of LL to rapidly fabricate microfluidic devices that can generate monodisperse microdroplets of user-defined composition on-demand. Our demonstration that this can be achieved using either lipids or surfactant to stabilise the water-oil interface, coupled with our ability to finely control the encapsulation and mixing of different components inside the droplets, supports the claim that our approach can be used for a wide range of applications. This powerful notion is underlined by the compatibility of DRF with a range of different solvents (See Table S1, Supporting Information) and our ability to initiate simple reactions inside droplets. Combining these factors indicates that more complex chemistries could be realised, particularly if the number of aqueous inlets is increased. The ability to control the concentration and level of mixing of components inside our droplets suggests that an array of chemically and physically independent bioreactors could be engineered. Alternatively, higher order function across these compartments could be achieved by connecting them via membrane active channels and pores. These proteins can be regulated using blockers ${ }^{31}$ and have been shown to mediate the communication and exchange of materials between compartments and facilitate reaction cascades. ${ }^{32}$ Moreover, it has also been shown that droplets assembled in this fashion can also be reversibly switched from an activated and inactivated state, ${ }^{33}$ or from an independent to an interconnected state using external light triggers. ${ }^{34,35}$ While the

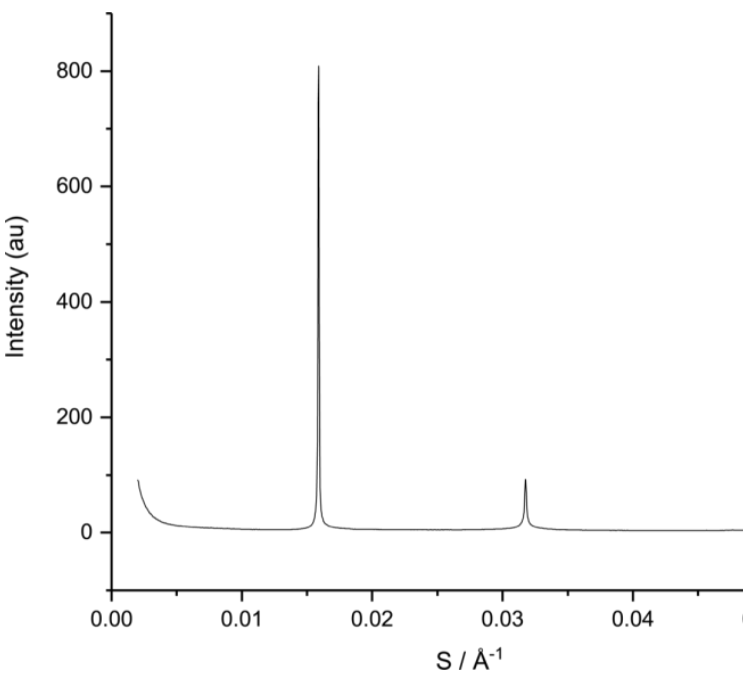

Fig. 5 Synchrotron small angle X-ray diffraction (SAXS) patterns from an aqueous DOPC sample (30 wt\%) pumped into a channel in the DRF system at $21^{\circ} \mathrm{C}$. smallest channels created using our approach are ca. 6x larger and significantly less linear than those that can be engineered using conventional photolithography (See supplementary information), ${ }^{36}$ this level of resolution is often not required by the end user and can be improved by optimizing the cutting process and by using a laser with a smaller spot size. The use of acrylic in combination with DRF also presents enhanced solvent compatibility compared to PDMS, simplifies bonding, creates less wastage and does not require any significant curing time, meaning that chips can be fabricated and used almost immediately. This is particularly desirable when optimizing new microfluidic technologies, as devices can be designed and assembled on-the-fly, without waiting for a new photomask and with minimum disruption to experimental work. This fast turnaround time between device iterations is also boosted by the fact that multiple devices can be engineered simultaneously across a typically larger area than is offered by a standard wafer. Furthermore, our results also indicate that devices engineered using LL are also much more resistant to high pressure compared to PDMS devices despite being thinner. The significance of this slim profile is perhaps best illustrated when using our microfluidic devices in combination with a synchrotron light-source. In this context, the devices produced using LL offered high transparency (low attenuation), low level of scattering and resistance to X-ray radiation without the need for thin X-ray compatible 'windows' as typically employed in other devices. ${ }^{37}$ This presents huge advantages in terms of device fabrication over existing approaches, such as the use of silicon nitride windows on Kapton foils, ${ }^{38,39}$ for example, or the use of polystyrene windows on chips cast from UV photocurable adhesives. ${ }^{40}$ Our approach, therefore, represents the ideal for investigators wishing to adopt a rapid and low-cost microfluidic platform for SAX/WAX studies.

These features highlight the huge potential of LL as a viable method for microfluidic device fabrication, particularly given how well it could lend itself to assembling multi-layered platforms. Given the existing compatibility of DRF with microelectronics, ${ }^{16,17}$ the next challenges will be to incorporate fully working valves and to develop new, open-source interconnects to economize device fabrication even further. Realizing these goals will enable the development of complex fluidic circuit boards that could be designed for almost any application.

\section{CONCLUSIONS}

In summary, by assembling droplet generators and micromixers capable of sustaining chemical reactions we have demonstrated LL to be a potent and versatile method that could be easily adapted to many applications. Our method is a simple, rapid and low-cost solution to a problem faced by all laboratories entering the field of microfluidics, which leads us to believe that LL will open up microfluidics for interdisciplinary research across the life sciences.

\section{ASSOCIATED CONTENT}

\section{Supporting Information}

The Supporting Information is available free of charge on the ACS Publications website.

\section{AUTHOR INFORMATION}

\section{Corresponding Author}

*Email: t.trantidou@imperial.ac.uk 
*Email: m.friddin@imperial.ac.uk

*Email: o.ces@imperial.ac.uk

\section{ORCID}

Tatiana Trantidou: 0000-0001-6784-2665

Mark S. Friddin: 0000-0003-4421-8792

Nicholas J. Brooks: 0000-0002-1346-9559

Oscar Ces: 0000-0002-6418-5644

\section{ACKNOWLEDGMENT}

The authors would like to thank Lee Tooley and Tony Meredith for their assistance with laser cutting. This work was supported by the EPSRC via grants EP/J017566/1 and EP/K038648/1. We acknowledge Diamond Light Source for provision of synchrotron beamtime (SM16024), and we would like to thank Andy Smith for assistance in operating beamline I22.

\section{REFERENCES}

(1) Huebner, A.; Sharma, S.; Srisa-Art, M.; Hollfelder, F.; Edel, J. B.; deMello, A. J. Lab on a Chip 2008, 8, 1244-1254.

(2) David J. Beebe; Glennys A. Mensing, a.; Walker, G. M. Annual Review of Biomedical Engineering 2002, 4, 261-286.

(3) and, Y. X.; Whitesides, G. M. Annual Review of Materials Science 1998, 28, 153-184.

(4) Xia, Y.; Whitesides, G. M. Angewandte Chemie 1998, 110, 568-594.

(5) Johnston, I. D.; McCluskey, D. K.; Tan, C. K. L.; Tracey, M. C. Journal of Micromechanics and Microengineering 2014, 24, 035017.

(6) Macdonald, N. P.; Cabot, J. M.; Smejkal, P.; Guijt, R. M.; Paull, B.; Breadmore, M. C. Analytical Chemistry 2017, 89, 38583866.

(7) Au, A. K.; Huynh, W.; Horowitz, L. F.; Folch, A. Angewandte Chemie International Edition 2016, 55, 3862-3881.

(8) Ho, C. M. B.; Ng, S. H.; Li, K. H. H.; Yoon, Y.-J. Lab on a Chip 2015, 15, 3627-3637.

(9) Waheed, S.; Cabot, J. M.; Macdonald, N. P.; Lewis, T.; Guijt, R. M.; Paull, B.; Breadmore, M. C. Lab on a Chip 2016, 16, 19932013.

(10) Stöhr, U.; Dohse, A.; Hoppe, P.; Thomas, M.; Kadel, K.; Klages, C. P.; Reinecke, H. Plasma Processes Polymers 2009, 6, 228233.

(11) Zhang, L.; Wang, W.; Ju, X.-J.; Xie, R.; Liu, Z.; Chu, L.-Y. RSC Advances 2015, 5, 5638-5646.

(12) Stephan, K.; Pittet, P.; Renaud, L.; Kleimann, P.; Morin, P.; Ouaini, N.; Ferrigno, R. Journal of Micromechanics and Microengineering 2007, 17, N69.

(13) Sandison, M. E.; Morgan, H. Journal of Micromechanics and Microengineering 2005, 15, S139.

(14) Leech, P. W.; Wu, N.; Zhu, Y. Journal of Micromechanics and Microengineering 2009, 19, 065019.

(15) Paul, D.; Saias, L.; Pedinotti, J.-C.; Chabert, M.; Magnifico, S.; Pallandre, A.; Lambert, B. D.; Houdayer, C.; Brugg, B.; Peyrin, J.M.; Viovy, J.-L. Biomicrofluidics 2011, 5, 024102.

(16) Moschou, D.; Vourdas, N.; Kokkoris, G.; Papadakis, G.; Parthenios, J.; Chatzandroulis, S.; Tserepi, A. Sensors and Actuators B: Chemical 2014, 199, 470-478.

(17) Vulto, P.; Huesgen, T.; Albrecht, B.; Urban, G. Journal of Micromechanics and Microengineering 2009, 19, 077001.

(18) Tsai, Y.-C.; Jen, H.-P.; Lin, K.-W.; Hsieh, Y.-Z. Journal of Chromatography A 2006, 1111, 267-271.

(19) Vulto, P.; Glade, N.; Altomare, L.; Bablet, J.; Medoro, G.; Leonardi, A.; Romani, A.; Chartier, I.; Manaresi, N.; Tartagni, M. SPECIAL PUBLICATION-ROYAL SOCIETY OF CHEMISTRY 2004, 297, 43-45.

(20) Guijt, R. M.; Candish, E.; Breadmore, M. C. Electrophoresis 2009, 30, 4219-4224.

(21) Jahn, A.; Stavis, S. M.; Hong, J. S.; Vreeland, W. N.; DeVoe, D. L.; Gaitan, M. ACS nano 2010, 4, 2077-2087.

(22) Jahn, A.; Vreeland, W. N.; DeVoe, D. L.; Locascio, L. E.; Gaitan, M. Langmuir 2007, 23, 6289-6293.
(23) Trantidou, T.; Friddin, M.; Elani, Y.; Brooks, N. J.; Law, R. V.; Seddon, J. M.; Ces, O. ACS Nano 2017, 11, 6549-6565.

(24) Venkatesan, G. A.; Lee, J.; Farimani, A. B.; Heiranian, M.; Collier, C. P.; Aluru, N. R.; Sarles, S. A. Langmuir 2015, 31, 1288312893.

(25) Asami, H.; Google Patents, 2001.

(26) Kang, S.-F.; Liao, C.-H.; Po, S.-T. Chemosphere 2000, 41, 1287-1294.

(27) El-Sharkawy, E.; Soliman, A. Y.; Al-Amer, K. M. Journal of Colloid and Interface Science 2007, 310, 498-508.

(28) Olajire, A.; Olajide, A. J Phys Chem Biophys 2014, 4, 136.

(29) Martin, H. P.; Brooks, N. J.; Seddon, J. M.; Luckham, P. F.; Terrill, N. J.; Kowalski, A. J.; Cabral, J. T. Soft Matter 2016, 12, 1750-1758.

(30) Holme, M. N.; Rana, S.; Barriga, H. M. G.; Kauscher, U.; Brooks, N. J.; Stevens, M. M. ACS Nano 2018, 12, 8197-8207.

(31) Thomas, J. M.; Friddin, M. S.; Ces, O.; Elani, Y. Chemical Communications 2017.

(32) Elani, Y.; Law, R. V.; Ces, O. Nat Commun 2014, 5.

(33) Chan, C. L.; Bolognesi, G.; Bhandarkar, A.; Friddin, M. S.; Brooks, N. J.; Seddon, J. M.; Law, R. V.; Barter, L. M.; Ces, O. Lab on a Chip 2016, 16, 4621-4627.

(34) Booth, M. J.; Restrepo Schild, V.; Box, S. J.; Bayley, H. Scientific Reports 2017, 7, 9315.

(35) Booth, M. J.; Schild, V. R.; Graham, A. D.; Olof, S. N.; Bayley, H. Science Advances 2016, 2.

(36) Trantidou, T.; Regoutz, A.; Voon, X. N.; Payne, D. J.; Ces, O. Sensors and Actuators B: Chemical 2018, 267, 34-41.

(37) Ghazal, A.; Lafleur, J. P.; Mortensen, K.; Kutter, J. P.; Arleth, L.; Jensen, G. V. Lab on a Chip 2016, 16, 4263-4295.

(38) Weinhausen, B.; Köster, S. Lab on a Chip 2013, 13, 212-215.

(39) Murray, T. D.; Lyubimov, A. Y.; Ogata, C. M.; Vo, H.; Uervirojnangkoorn, M.; Brunger, A. T.; Berger, J. M. Acta Crystallographica Section D: Biological Crystallography 2015, 71, 1987-1997.

(40) Ghazal, A.; Gontsarik, M.; Kutter, J. P.; Lafleur, J. P.; Labrador, A.; Mortensen, K.; Yaghmur, A. Journal of Applied Crystallography 2016, 49, 2005-2014.

\section{Insert Table of Contents artwork here}

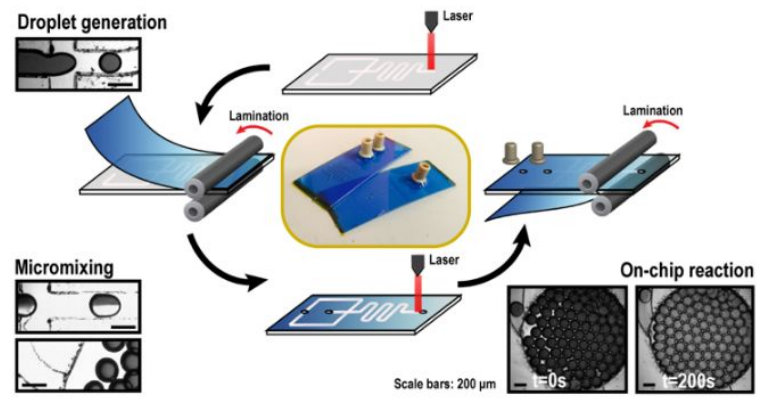

